\title{
Individual T/R Module Characterisation of the TerraSAR-X Active Phased Array Antenna by Calibration Pulse Sequences with Orthogonal Codes
}

\author{
Benjamin Bräutigam, Marco Schwerdt, Markus Bachmann \\ Microwaves and Radar Institute of the German Aerospace Center (DLR) \\ Oberpfaffenhofen, P.O.-Box 1116, D-82230 Weßling, Germany \\ Email: Benjamin.Braeutigam@dlr.de
}

\author{
Martin Stangl \\ EADS Astrium GmbH Friedrichshafen \\ 88039 Friedrichshafen, Germany
}

\begin{abstract}
TerraSAR-X is a high resolution synthetic aperture radar (SAR) satellite due for launch in 2007. Its active phased array X-Band antenna hosts 384 transmit/receive modules controlling the beam steering in azimuth and elevation direction. Precise modelling of the antenna is only possible if the actual characteristics of each individual transmit/receive module are known. TerraSAR-X has been equipped with an innovative characterisation mode based on the so-called PN Gating method. Individual and simultaneous characterisation of all transmit/receive modules is realised under most realistic conditions with the same power loads like in the nominal mode. This paper shows the results of PN Gating measurements on a satellite SAR system.
\end{abstract}

Index Terms-TerraSAR-X, Internal Calibration, Transmit/Receive Modules, Phased Array Antenna, Synthetic Aperture Radar (SAR).

\section{INTRODUCTION}

The first German SAR satellite for commercial and scientific applications, TerraSAR-X, will start its operational phase in 2007. TerraSAR-X is a flexible $X$-Band SAR operating in Stripmap, Spotlight, ScanSAR, and additional experimental modes [1]. For the various antenna beams, an active phased array antenna electronically shapes the patterns. The array consists of 384 radiating sub-arrays for horizontal and vertical polarisation arranged in a matrix of $N=12$ panels with $M=32$ rows. Active transmit/receive (T/R) modules individually adjust the array elements in gain and phase for tapering and steering of the antenna pattern in azimuth and elevation direction [2],[3]. An antenna model mathematically describes the beam shapes from detailed pattern measurements of the antenna sub-arrays combined with the applied gain and phase excitations.

Even though the TerraSAR-X X-band front-end (XFE) with a large number of T/R modules (TRMs) and radiators is designed to be insensitive to contingencies like those of individually failed or drifted modules, it is necessary to detect such failures and characterise the TRMs.

In the module stepping mode of the ENVISAT ASAR instrument [4], individual measurements on the excitation coefficients of the TRMs are only possible if all modules except the one being characterised are switched off. However, individual

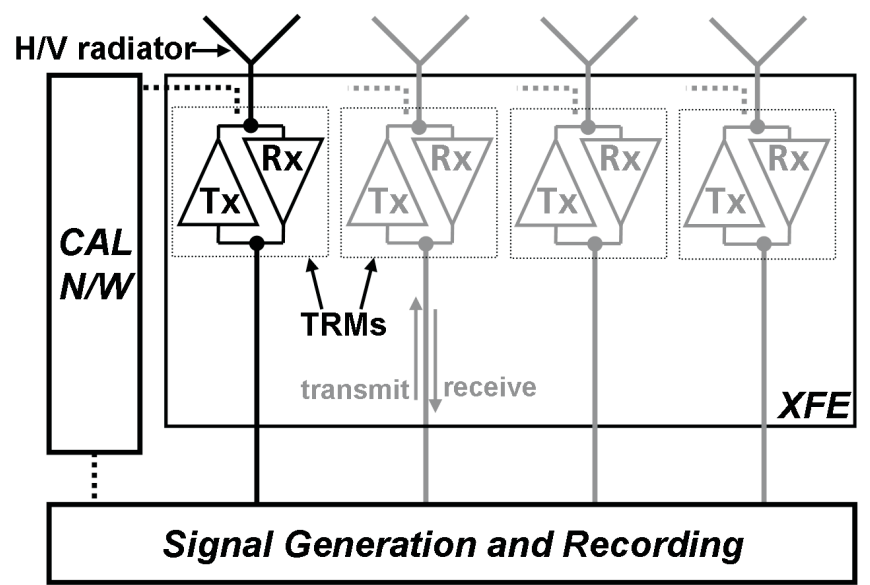

Fig. 1. X-Band Front-End (XFE) of TerraSAR-X Radar with 4 of 384 Transmit/Receive Modules (TRMs). The calibration signal is routed via couplers at the TRMs and the calibration network (CAL N/W).

measurements of TRMs under most realistic conditions require the same power loads like in the nominal mode with all TRMs operating. This paper shows the advantages of individual TRM characterisation with the more efficient PN Gating method [5] in the frame of TerraSAR-X on-ground measurements. For the first time, this method is applied to a real active phased array SAR antenna for performance monitoring.

\section{Internal Calibration Pulse Sequences with ORTHOGONAL CODES}

\section{A. Internal Calibration Architecture}

For calibrating and monitoring the instrument stability, the radar instrument of TerraSAR-X features an internal calibration facility coupling into an additional port of each TRM as shown in Fig. 1. Calibration pulses are routed through the XFE to characterise critical elements of the transmit (TX) and receive (RX) path. The acquired signals can only be measured at the composite ports of the distribution networks [6]. In orbit, absolute power level degradation is calibrated via external targets like transponders or corner reflectors [7]. Thus, only relative characterisation results are of interest. 


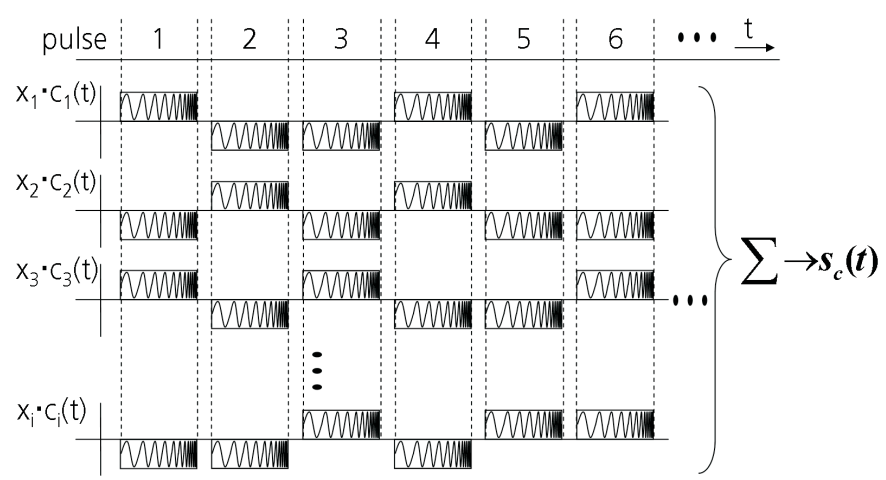

Fig. 2. Superposition of signals of all TRMs. Each signal is scrambled by its own code sequence applied from pulse to pulse.

\section{B. Estimation of Individual TRM Characteristics}

Apart from measuring the stability of the instrument it is necessary to retrieve information on the performance of individual TRMs. The tapering and steering of the antenna beam depends on the beam excitation coefficients defining gain and phase of the TRMs. The actual status of TRM setting has to be known, especially considering performance degradation or malfunction. Comparing telemetry data (e.g. voltage and temperature behaviour of the TRMs) to appropriated onground characterisation only provides limited information on the radar performance. Direct RF measurements of individual TRMs would only be possible if all modules except the one being characterised are switched off. This so-called module stepping procedure - as used for the ENVISAT ASAR instrument - does not cover the actual status of operating modules due to different power supply loading in this mode.

A detailed analysis of individual TRMs within an active phased array antenna is based on the PN-Gating method developed at the German Aerospace Center (DLR) [5]. The name "PN Gating method" refers to the possibility of scrambling the TRM signals with a pseudo-noise (PN) code. The advantage of this technique is that individual TRMs are characterised while all modules are operating, i.e. a characterisation under the most realistic conditions. In this special characterisation mode the actual phase of each TRM is individually shifted by $\pm 90^{\circ}$ from pulse to pulse according to a defined code sequence $c_{m n}(t)$.

Hence, the total phase commanded for a transmit/receive module is the phase $\varphi_{m n}$ of its setting plus a shift by $90^{\circ}$. Consequently, the superposition of all TRM gains $a_{m n}$ and phases $\varphi_{m n}$ at the composite port of the distribution network yields the composite signal $s_{c}(t)$ as shown in Fig. 2

$$
s_{c}(t)=\sum_{m=0}^{M-1} \sum_{n=0}^{N-1} c_{m n}(t) \cdot a_{m n} e^{j \varphi_{m n}}+n_{m n}
$$

where $n_{m n}$ is the TRM inherent noise. Hence, to extract the information for one TRM the composite signal is correlated with the corresponding code sequence providing the estimated gain and phase setting of this module. By this correlation process the code modulation is removed and the complex correlation peak represents an estimated gain $\tilde{a}_{m n}$ and phase $\tilde{\varphi}_{m n}$ of the respective TRMs:

$$
\begin{gathered}
\tilde{x}_{m n}=s_{c} \otimes c_{m n}^{*} \\
\tilde{x}_{m n}=\int s_{c}(t) \cdot c_{m n}^{*}(t) d t=\tilde{a}_{m n} e^{j \tilde{\varphi}_{m n}}
\end{gathered}
$$

Simulations have shown the impact of different code types on the quality of the correlation process [6], [5]. The Walsh code has proven its robustness for the TerraSAR-X system showing the best results compared to other code types like pseudo-noise gating. Orthogonal Walsh codes derived from Hadamard matrices separate each code from another. To keep the time and data volume low the applied code length $l$ shall be as short as possible. The number of TRMs restricts the minimum code length

$$
l=2^{i} \geq N \cdot M
$$

with $M$ being the number of array rows and $N$ the number of array columns. The PN Gating mode of TerraSAR-X can be executed as three basic performance checks:

- module level with minimum code length of 512 bits for 384 modules,

- panel level with minimum code length of 16 bits for 12 panels,

- row level with minimum code length of 32 bits for 32 rows.

All 384 TRMs are characterised simultaneously in module level. For row level all modules of one row are assigned to one code, respectively, resulting in 32 different codes. For instance, the result of the first row is the average over the first row of all 12 panels. Thus, the row level type provides 32 estimation results of all rows representing the elevation settings of the antenna. The same accounts for panel level yielding 12 estimation results for 12 panels. Panel level means averaging over all rows of one panel, i.e. in elevation. The final results describe the behaviour of the antenna azimuth setting. The TerraSAR-X antenna beams are realised by applying rowwise excitation settings for elevation steering and panel-wise excitation settings for azimuth steering. Thus, panel and row level performance checks serve for characterisation of the antenna beam settings.

\section{INDIVIDUAL TRM CHARACTERISATION FOR TERRASAR-X}

\section{A. Antenna Performance Monitoring}

Several cases of performance degradation have been studied:

- single TRM failure,

- malfunction of half a panel (16 TRMs) in a tapered beam,

- gain and phase degradation of several TRMs.

The checks were executed as module level on the TerraSAR-X antenna with 384 TRMs and compared to previously acquired reference sets without malfunction. Each test run provides the estimated actual gain and phase setting of each TRM, both for TX and RX. 


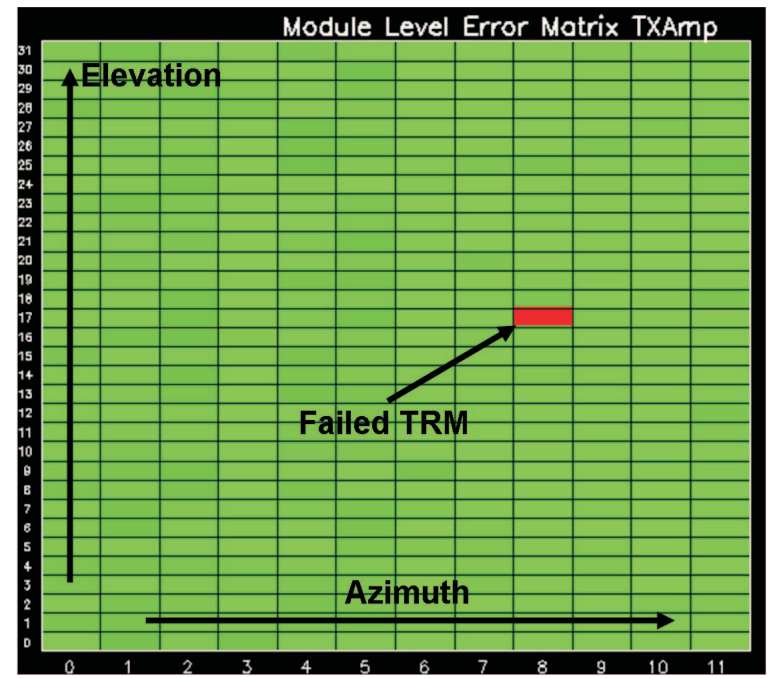

Fig. 3. Error Matrix of TerraSAR-X antenna. TX gain estimation derived from module level check with one TRM switched off at position 8/17.

1) TRM Failure from Module Level Check: In the instrument configuration one TRM was switched off. The results of a module level check are plotted into an error matrix system representing the antenna TRM configuration of TerraSAR$\mathrm{X}$. Fig. 3 shows the estimated gains in TX (the same exists for RX). High power levels are green-coloured, low power levels are red-coloured indicating module degradation. The evaluation shows a significant decrease in gain for one TRM. As each code assigns to an individual TRM, the position of the affected module can be easily retrieved. The position of the detected failed element is at the expected place conform to the assigned code at column 8 and row 17 .

2) Panel Malfunction in a Tapered Beam: Half a panel (16 TRMs) was switched off whereas the antenna was excited with a gain taper in elevation (row) direction. The commanded RX taper in elevation applied to all panels is drawn as a solid line in Fig. 4. From module level check individual row excitations of every panel can be measured. The estimated excitations of the individual panels are compared to the commanded ones. The estimated RX gain of Panel 0 is plotted as a dashed line. It matches the commanded beam taper very well. Accordingly, the RX gain characteristics (dotted line) of Panel 10 correspond to the expected values, too. The first 16 TRMs of Panel 10 yield only noise signal as these TRMs were switched off.

3) Gain and Phase Estimation: For accuracy check of the estimated excitations several TRMs were commanded with different excitation coefficients in gain and phase compared to boresight operation. Panels 8 and 9 were commanded with a shift of $+45^{\circ}$ in TX phase, while panels 2 and 3 were attenuated by $2.3 \mathrm{~dB}$ for RX. All other panels had nominal settings of $0 \mathrm{~dB}$ attenuation and $0^{\circ}$ phase in TX and RX.

Fig. 5 shows all TRM characteristics for TX and RX behaviour of the antenna. After averaging over all elements of one panel the estimated TX phase of panels 8 and 9 was $+45^{\circ}$

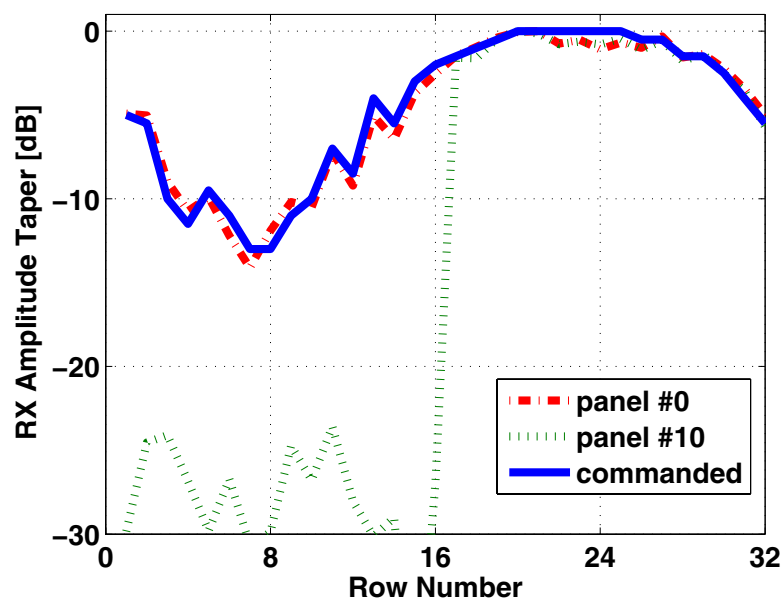

Fig. 4. Commanded and measured beam taper on panel 0 and panel 10 of TerraSAR-X antenna. The first 16 TRMs of panel 10 were switched off.
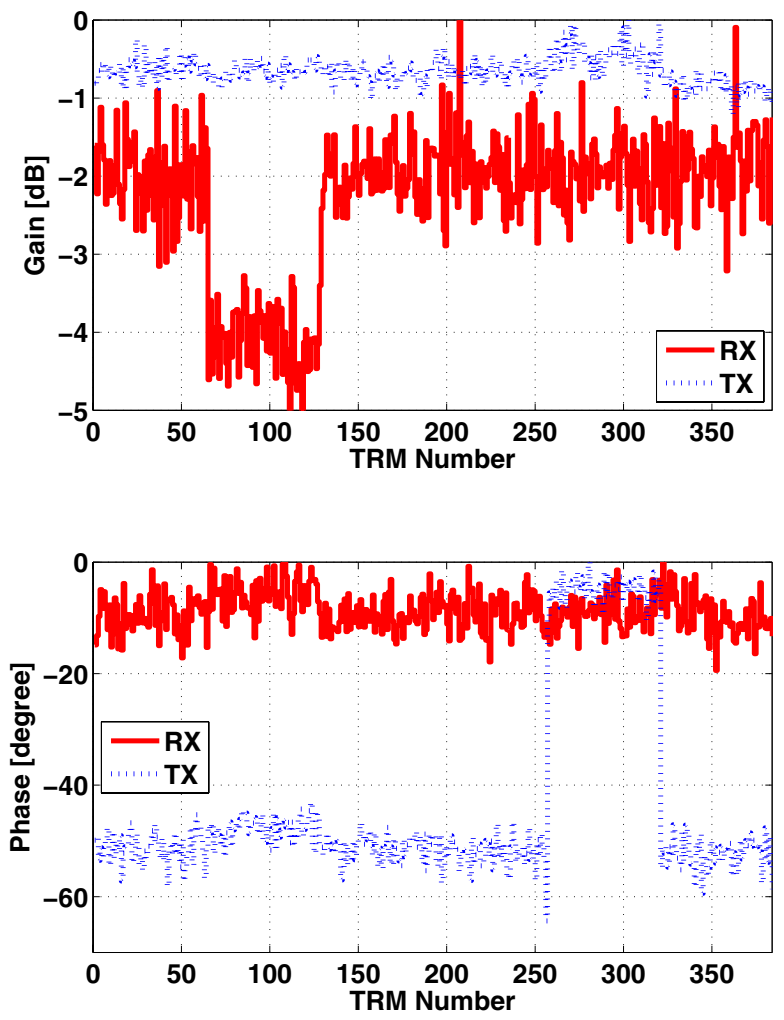

Fig. 5. Module level measurements of antenna in TX and RX. Each data set is referenced to its maximum value. Above - Gain degradation of $-2.4 \mathrm{~dB}$ in RX for TRMs 64 to 127 corresponding to panels 2 and 3. Below - Phase degradation of $+45^{\circ}$ at TRMs 256 to 319 corresponding to panels 8 and 9 . 
TABLE I

STANDARD DEVIATION OF REPEATED MEASUREMENTS

\begin{tabular}{lcccc}
\hline Repetition & TX Gain & RX Gain & TX Phase & RX Phase \\
\hline$\# 1$ & $0.16 \mathrm{~dB}$ & $0.38 \mathrm{~dB}$ & $2.0^{\circ}$ & $2.6^{\circ}$ \\
$\# 2$ & $0.10 \mathrm{~dB}$ & $0.27 \mathrm{~dB}$ & $1.4^{\circ}$ & $2.0^{\circ}$ \\
$\# 3$ & $0.09 \mathrm{~dB}$ & $0.24 \mathrm{~dB}$ & $1.1^{\circ}$ & $1.6^{\circ}$ \\
\hline \multirow{5}{c}{ Panel Level } \\
\hline$\# 1$ & $0.02 \mathrm{~dB}$ & $0.17 \mathrm{~dB}$ & $1.1^{\circ}$ & $0.7^{\circ}$ \\
$\# 2$ & $0.02 \mathrm{~dB}$ & $0.04 \mathrm{~dB}$ & $0.4^{\circ}$ & $0.1^{\circ}$ \\
$\# 3$ & $0.01 \mathrm{~dB}$ & $0.02 \mathrm{~dB}$ & $0.3^{\circ}$ & $0.3^{\circ}$ \\
\hline \multirow{5}{c}{ Row Level } \\
\hline 1 & $0.03 \mathrm{~dB}$ & $0.06 \mathrm{~dB}$ & $0.6^{\circ}$ & $0.5^{\circ}$ \\
$\# 2$ & $0.02 \mathrm{~dB}$ & $0.05 \mathrm{~dB}$ & $0.4^{\circ}$ & $0.4^{\circ}$ \\
$\# 3$ & $0.02 \mathrm{~dB}$ & $0.06 \mathrm{~dB}$ & $0.4^{\circ}$ & $0.3^{\circ}$ \\
\hline
\end{tabular}

as configured. The estimated RX gain of panels 2 and 3 results in $-2.4 \mathrm{~dB}$, only deviating by $0.1 \mathrm{~dB}$ from the commanded value.

\section{B. Measurement Accuracy}

During on-ground testing reference measurements have been repeated three times per module, row, and panel level checks in a time frame of several weeks. Each acquired data set has been compared to the initial one. The standard deviation was calculated from TRM characterisation results compared to the initial reference measurement. Table I lists the standard deviation of repeated measurements for different level types. With module level checks the standard deviation of estimated beam excitations of the TRMs stayed below $0.16 \mathrm{~dB}$ and $2^{\circ}$ for TX and below $0.38 \mathrm{~dB}$ and $2.6^{\circ}$ for RX. The accuracy in $\mathrm{TX}$ is better than for RX as the signal-to-noise ratio of the TX calibration signal is $8 \mathrm{~dB}$ higher. Module level checks provide precise estimation results on the degradation of single TRMs.

Panel level checks perform an estimation of the azimuth behaviour as all TRMs of one panel have the same code setting. In row level measurements all 12 TRMs per row have the same setting, i.e. the elevation behaviour is characterised. The panel and row level tests agree almost perfect to each other as they already contain an averaging over several modules. Estimated excitations are usually in the range of smaller than $0.1 \mathrm{~dB}$ in gain and better than $1^{\circ}$ in phase. The beam steering is realised by exciting the antenna in azimuth and elevation. Performing two consecutive measurements for $M$ rows and $N$ panels is not only a more effective way of characterising the antenna pattern. It also yields very accurate antenna beam estimations of the applied TRM settings for elevation and azimuth steering.

\section{CONCLUSION}

The PN Gating method allows operating all individual transmit/receive modules under most realistic conditions with the advantage that all modules can be characterised simultaneously. During on-ground characterisation of the TerraSAR-X radar instrument this technique has established as a crucial diagnostic tool for functional checks as well as TRM drift and failure monitoring.

The flexibility of the PN Gating method refers to different level checks possible on module, panel, and row-wise measurements. Module level checks provide precise estimations of TRM characteristics detecting performance degradation or module failure of individual TRMs. Panel and row level measurement represent the actual antenna excitation in elevation and azimuth. Additionally, they consume less time and data volume. The results from repeated measurements of the same instrument and antenna conditions prove the high measurement accuracy of the PN Gating technique. The repeated measurements were in a time frame of weeks. Still, the accordance of the estimation results to each other is almost perfect.

For the first time ever, this innovative method will be applied in a satellite environment during the TerraSAR-X mission. The fast and realistic estimation of individual TRM excitation during the lifetime of the instrument will be a valuable support for in-orbit characterisation and instrument performance monitoring. In case of contingencies like degraded or failed modules, these modules can be detected. The measured excitation can be fed into the antenna model for optimisation of the remaining settings [8]. This ensures further good instrument performance in spite of degraded transmit/receive modules. The technique described in this paper is also applicable for characterisation and calibration of other advanced sensor systems coping with active phased array antennas.

\section{REFERENCES}

[1] S. Buckreuss, W. Balzer, P. Mühlbauer, R. Werninghaus, and W. Pitz, "The TerraSAR-X Satellite Project," in IEEE: 25th International Geoscience And Remote Sensing Symposium, Toulouse, Frankreich, 2003.

[2] W. Pitz, "The TerraSAR-X Satellite," in 6th European Conference on Synthetic Aperture Radar, Dresden, Germany, 2006.

[3] M. Wahl, M. Adolph, K. Biller, U. Hackenberg, R. Rieger, B. Schweizer, B. Adelseck, H. Brugger, and M. Loercher, "High Precision T/R Module for SAR Earth Observation," in 6th European Conference on Synthetic Aperture Radar, Dresden, Germany, 2006.

[4] C. Buck, "Asar instrument gain monitoring," ESTEC, European Space Agency (ESA), ENVISAT ASAR Calibration Review (ECR), 2002.

[5] D. Hounam, M. Schwerdt, and M. Zink, "Active Antenna Module Characterisation by Pseudo-Noise Gating," in 25th ESA Antenna Workshop on Satellite Antenna Technology, Noordwijk, Netherlands, 2002.

[6] M. Schwerdt, D. Hounam, J. L. Alvarez-Pérez, and T. Molkenthin, "The Calibration Concept of TerraSAR-X, a Multiple Mode High Resolution SAR," Canadian Journal of Remote Sensing, pp. 30-36, February 2005.

[7] B. Bräutigam, M. Schwerdt, and M. Bachmann, "The External Calibration of TerraSAR-X, a Multiple Mode SAR-System," in 6th European Conference on Synthetic Aperture Radar, Dresden, Germany, 2006.

[8] M. Bachmann, M. Schwerdt, B. Bräutigam, B. Grafmüller, A. Herschlein, and J. L. Alvarez-Pérez, "The TerraSAR-X Antenna Model Approach," in 2nd International ITG Conference on Antennas, Munich, Germany, 2007. 